\title{
Spinal Anesthesia for Emergent Testicular Torsion Surgery in a Pediatric Patient with Spinal Muscular Atrophy Type-II
}

\author{
Hande Gurbuz Aytuluk ${ }^{1 *}$ and Hulya Topcü \\ ${ }^{1}$ Department of Anesthesiology and Reanimation, University of Health Sciences, Derince Training and \\ Research Hospital, Turkey \\ ${ }^{2}$ Department of Anesthesiology and Reanimation, Hitit University, Erol Olcok Training and Research Hospital, \\ Turkey
}

*Corresponding author: Hande Gurbuz Aytuluk, Derince Training and Research Hospital, Department of Anesthesiology and Reanimation, Kocaeli, Turkey

\begin{abstract}
Spinal muscular atrophy (SMA) is one of the most common inherited diseases in children. The clinical symptoms of this neuromuscular disease vary widely from muscle weakness to death according to the onset of symptoms. There is no specific treatment for the disease. There are many risks for anesthesia, as these patients usually have skeletal deformities, increased sensitivity to opioids and neuromuscular blockers, and pulmonary disease secondary to respiratory muscle dysfunction. There is no evidence-based advice or guideline for the management of anesthesia in SMA patients and that most of the literature is based upon case reports. In this report, a 13-year-old pediatric patient who underwent an emergent surgery for testicular torsion under neuraxial anesthesia is presented.
\end{abstract}

\section{Keywords}

Anesthesia, Spinal muscular atrophy, Spinal neuromuscular diseases, Spinal muscular atrophies of childhood

\section{Introduction}

Spinal muscular atrophy (SMA), which has an autosomal recessive inheritance, is one of the most common inherited diseases in children. The main pathology is the degeneration of the spinal motor neurons, herewith it presents with progressive symmetrical muscle weakness and increasing hyporeflexia, especially in upper extremities [1]. Sensory nerve functions may also be affected in severely ill patients, however cognitive and emotional functions are always intact in SMA patients
[1]. The clinical symptoms and the progression rate of this neuromuscular disease vary widely from muscle weakness to death according to the onset of symptoms. There is no specific treatment for the disease, however functional status can be preserved for a very long time with a well-care and physical rehabilitation.

Patients with SMA often need anesthesia for various interventional procedures. The risks for anesthesia vary in relation with the type of the disease. In this report, a 13-year-old pediatric patient who underwent an emergent surgery for testicular torsion under neuraxial anesthesia is presented.

\section{Case Report}

A 13-year-old boy $1.50 \mathrm{mt}$ in height and $40 \mathrm{~kg}$ in weight, underwent emergent surgery with a diagnosis of testicular torsion. It was learnt from his parents that he was diagnosed as SMA type- 2 in the infantile period. He used no medications for the disease, and he was included in a regular physical rehabilitation program. He had difficulty with standing on his own, however he could sit without help. Respiratory and cardiac functions, and laboratory analyses were evaluated as normal in the preoperative examination. He had a mild thoracal kyphoscoliosis. Mouth opening, and neck movements were in normal range. Following obtaining the written informed consent from his parents, the patient was informed about the plan for regional

Citation: Aytuluk HG, Topcu H (2019) Spinal Anesthesia for Emergent Testicular Torsion Surgery in a Pediatric Patient with Spinal Muscular Atrophy Type-II. Int J Anesthetic Anesthesiol 6:087. doi. org/10.23937/2377-4630/1410087

Accepted: April 03, 2019: Published: April 05, 2019

Copyright: (c) 2019 Aytuluk HG. This is an open-access article distributed under the terms of the Creative Commons Attribution License, which permits unrestricted use, distribution, and reproduction in any medium, provided the original author and source are credited. 
anesthesia. The patient was fully cooperative and his assent for subarachnoid block was obtained. He was also informed about postoperative paresthesia.

No premedication was administered to the patient. With the patient in the sitting position, a spinal block was performed with a 25-gauge Quincke needle inserted in the L3/4 interspace. Following the free flow of clear cerebrospinal fluid, $0.5 \%$ hyperbaric bupivacaine (10 $\mathrm{mg}$ ) was injected, after which the patient was placed in the supine position. The patient received $1 \mathrm{mg}$ midazolam intravenous for sedation, and $2 \mathrm{ml} \mathrm{min}^{-1} \mathrm{O}_{2}$ via face mask. The surgery was started when the sensory level T7 (blocked to pinprick) was achieved. The right testis was totally necrosed that right orchidectomy was performed. The surgery was completed in about 1 hour uneventfully. Bromage score " 0 " was achieved at the $3^{\text {rd }}$ hour of the postoperative period. Oral paracetamol and ibuprofen were used for postoperative pain management. The patient was discharged from the hospital on the following day.

\section{Discussion}

The onset of the symptoms of SMA is associated with the severity, and progression of the disease. Spinal muscular atrophy can be assorted into 5 groups according to the age of onset of the symptoms [2]. SMA type 0 is the prenatal type, and usually fatal at birth. SMA type 1 (Werdnig-Hoffman disease) is the rapidly progressive infantile type SMA which is diagnosed before 6 months and had early onset of respiratory failure. SMA type 2 (Dubowitz type) is the intermediate SMA that is diagnosed before the $1^{\text {st }}$ age. These patients can sit- but cannot walk, and they can reach adulthood in a very good condition with a well medical care. SMA type 3 (Kugelberg Welander's disease) is the juvenile form of the disease, in which onset of the symptoms is in the late childhood period. These patients can walk with help. SMA type 4 is the adult form of the disease in which the onset of symptoms is in adulthood. Natural life span of the patients is not affected in SMA types 3 and 4.

Simple interventions (muscle biopsy for example) or major surgical procedures (vertebral surgery for example) are sometimes required in patients with SMA. The main perioperative problem is pulmonary complications and prolonged mechanical ventilation. There are many risks for anesthesia, as these patients usually have skeletal deformities, increased sensitivity to opioids and neuromuscular blockers, and pulmonary disease secondary to respiratory muscle dysfunction. There is no evidence-based advice or guideline for the management of anesthesia in SMA patients and that most of the literature is based upon case reports. There are many examples of general and regional anesthesia resulting in success or failure in these patients. None of them is the best technique, so anesthetic management must be individualized according to the clinical symptoms in SMA patients.

For airway management, tracheal intubation can be extremely difficult because of the limited mobility of the cervical spine or reduced mouth opening due to joint contractures. Succinylcholine is adviced to be avoided in patients with neuromuscular diseases because of the risk of hyperkalemia, rhabdomyolysis, and malignant hyperthermia, however there is no report of any adverse events with succinylcholine in SMA patients. There is an increased sensitivity to nondepolarizing agents due to the decrease of choline acetyltransferase and acetylcholinesterase levels, so it would be better to avoid muscle relaxants if possible [3]. If it is necessary to use muscle relaxants, sugammadex is a good option for the reversal [3]. Volatile agents have been used successfully in the anesthetic management of SMA patients. Because these patients are prone to respiratory depression, short-acting opioids such as remifentanil can be preferred, or even opioid-free anesthesia can be considered.

Regional anesthesia has been used successfully in SMA patients and it is a safe option in suitable cases [4]. However, the neuraxial blocks can be technically difficult in many cases because of vertebral structural abnormalities including severe scoliosis, and the distribution of the local anesthetic may be inadequate [5]. Although pediatric regional anesthesia is recommended in guidelines, it still remains as a main concern for many physicians [6]. The need for deep sedation or general anesthesia while performing regional anesthesia in non-cooperative small children and the apprehension for adjusting accidentally overdose of the local anesthetic drugs discourages the majority of anesthesiologists. In the present case the patient was fully cooperative, and scoliosis was not severe, so that performing spinal anesthesia was not challenging. Nonetheless, regional anesthesia may be performed safely even in small children with SMA.

\section{Conclusion}

Anesthetic management must be individualized in spinal muscular atrophy patients according to variable clinical presentation.

\section{Acknowledgement}

This report was presented in the $3^{\text {rd }}$ Palandoken Anesthesia Days, Pediatric Anesthesia, 2018, Erzurum, Turkey as an oral presentation.

\section{References}

1. D'Amico A, Mercuri E, Tiziano FD, Bertini E (2011) Spinal muscular atrophy. Orphanet J Rare Dis 6: 71.

2. Islander G (2013) Anesthesia and spinal muscle atrophy. Paediatr Anaesth 23: 804-816.

3. Hatipoglu Z, Gulec E, Turktan M, Tarak N, Unlugenc H (2015) Successful recurarization with sugammadex in caesarean section patient with spinal muscular atrophy: A 
case report. Turkiye Klinikleri J Case Rep 23: 355-358.

4. Weston LA, DiFazio CA (1996) Labor analgesia and anesthesia in a patient with spinal muscular atrophy and vocal cord paralysis. A rare and unusual case report. Reg Anesth 21: 350-354.

5. Haché M, Swoboda KJ, Sethna N, Farrow-Gillespie A, Khandji A, et al. (2016) Intrathecal injections in children with spinal muscular atrophy: Nusinersen clinical trial experience. J Child Neurol 31: 899-906.

6. Ivani G, Suresh S, Ecoffey C, Bosenberg A, Lonnqvist PA, et al. (2015) The european society of regional anaesthesia and pain therapy and the american society of regional anesthesia and pain medicine joint committee practice advisory on controversial topics in pediatric regional anesthesia. Reg Anesth Pain Med 40: 526-532. 